\title{
Enhanced Chosen-Ciphertext Security and Applications
}

\author{
Dana Dachman-Soled ${ }^{1}$, Georg Fuchsbauer ${ }^{2}$, Payman Mohassel ${ }^{3}$, \\ and Adam O'Neill ${ }^{4}$ \\ 1 University of Maryland \\ danadach@ece.umd.edu \\ 2 Institute of Science and Technology Austria \\ georg. fuchsbauer@ist.ac.at \\ 3 University of Calgary \\ pmohasse@cpsc.ucalgary.ca \\ ${ }^{4}$ Georgetown University \\ adam@cs.georgetown.edu
}

\begin{abstract}
We introduce and study a new notion of enhanced chosenciphertext security (ECCA) for public-key encryption. Loosely speaking, in the ECCA security experiment, the decryption oracle provided to the adversary is augmented to return not only the output of the decryption algorithm on a queried ciphertext but also of a randomness-recovery algorithm associated to the scheme. Our results mainly concern the case where the randomness-recovery algorithm is efficient.

We provide constructions of ECCA-secure encryption from adaptive trapdoor functions as defined by Kiltz et al. (EUROCRYPT 2010), resulting in ECCA encryption from standard number-theoretic assumptions. We then give two applications of ECCA-secure encryption: (1) We use it as a unifying concept in showing equivalence of adaptive trapdoor functions and tag-based adaptive trapdoor functions, resolving an open question of Kiltz et al. (2) We show that ECCA-secure encryption can be used to securely realize an approach to public-key encryption with non-interactive opening (PKENO) originally suggested by Damgård and Thorbek (EUROCRYPT 2007), resulting in new and practical PKENO schemes quite different from those in prior work.

Our results demonstrate that ECCA security is of both practical and theoretical interest.
\end{abstract}

\section{Introduction}

This paper introduces and studies a new notion of security for public-key encryption (PKE) we call enhanced chosen-ciphertext security (ECCA). Besides being interesting in its own right, we find that ECCA security plays a fundamental role in contexts where randomness-recovering encryption (as discussed informally in e.g. 29]) is important, such as adaptive trapdoor functions [25] and PKE with non-interactive opening [15. We also believe ECCA will find further applications in the future. Below we describe our results concerning ECCA in more detail; for a pictorial summary, see Figure 1 . 


\subsection{ECCA Security Definition and Variants}

Recall that in the standard formulation of CCA security [30], the adversary, given a public key pk, must guess which of the two possible messages its challenge ciphertext $c$ encrypts, while being allowed to query a decryption oracle on any ciphertext $c^{\prime}$ different from $c$. Very informally, our "enhancement" is that the decryption oracle, when queried on a ciphertext $c^{\prime}$, returns not only the output of the decryption algorithm of the scheme run on $c^{\prime}$, but also of an associated randomness-recovery algorithm. This randomness-recovery algorithm, given sk and an honestly generated encryption $c$ of $m$ with coins $r$, is guaranteed to output some coins $r^{\prime}$ such that the encryption of $m$ with coins $r^{\prime}$ is also $c$. (However, like the decryption algorithm — which is only guaranteed to output the right message on honestly generated ciphertexts - its behavior on other, maliciously generated ciphertexts depends on its specification.)

Note that in general we do not require that $r=r^{\prime}$ above, but in the special case that this holds we say that the scheme is uniquely randomness-recovering. Looking ahead, our constructions of ECCA-secure PKE will be uniquely RR, but for some applications this is not strictly necessary as long as the scheme has perfect correctness (i.e., zero decryption error).

Our study of ECCA security is largely motivated by the related concept of randomness-recovering $(\mathrm{RR})$ encryption, in which case the randomness-recovery algorithm is efficient. Indeed, we show that not every CCA-secure RR encryption scheme is ECCA-secure (cf. Proposition 1). This means that in applications of RR encryption that require ECCA security, it may not be sufficient to use a scheme proven CCA-secure.

\subsection{Constructions of ECCA-Secure PKE}

ECCA-Secure PKE from Adaptive TDFs. The first standard-model construction of CCA-secure randomness-recovering PKE was achieved by Peikert and Waters [29], based on their new concept of "lossy" trapdoor functions (TDFs). A line of subsequent work 3125] focused on achieving CCA-secure PKE from progressively weaker assumptions on TDFs 1 This leads one to wonder whether these assumptions suffice for ECCA-secure RR PKE as well. Ideally, one would achieve ECCA-secure, uniquely RR PKE - the strongest form of randomness-recovery - based on adaptive TDFs, the weakest of these assumptions. (Intuitively, adaptivity is a form of CCA security for TDFs, asking that the TDF remain one-way even when the adversary may query an inversion oracle on points other than its challenge.) This is exactly what our results obtain.

Challenges and Techniques. Our construction is technically novel, as the construction of CCA-secure encryption from adaptive TDFs in the earlier work

\footnotetext{
${ }^{1}$ Wee 32 showed that a weaker notion of adaptivity for trapdoor relations suffices; however, as this is not an assumption on trapdoor functions it does not seem to yield RR encryption and won't be useful for our results.
} 
of 25. seems to be neither RR nor ECCA-secure (we achieve both, and moreover unique randomness recovery). Indeed, in the construction of [25] a general transform of [27] is used to convert a one-bit CCA-secure PKE from ATDFs to a multi-bit CCA-secure one. However, this transform does not seem to preserve either randomness-recovery nor ECCA-security of the one-bit scheme. Furthermore, the one-bit scheme of [25] — which works by re-sampling a domain point $x$ until the hardcore bit of $x$ equals the message - is not uniquely RR, since decryption does not recover the "thrown away" $x$ 's. (Note that the "naïve" one-bit scheme from ATDFs that simply XOR's the message bit with a hardcore bit of the ATDF is trivially malleable by flipping the last bit of a ciphertext and thus is not CCA-secure.)

We solve these problems via a novel application of detectable CCA (DCCA) security, introduced recently by Hohenberger et al. 24]. Informally, DCCA is defined relative to a "detecting" function $\mathcal{F}$ (which must satisfy some definitions) that determines whether two ciphertexts are related; in the DCCA experiment, the adversary is not allowed to ask for decryptions of ciphertexts related to the challenge ciphertext according to $\mathcal{F}$. The work of 24 gives a transform from any DCCA-secure PKE to a CCA-secure encryption one. In particular, bit-bybit encryption using a 1-bit CCA-secure encryption scheme is DCCA-secure, thus encompassing the earlier work of [27]. Our novelty is that we construct a DCCA-secure scheme from ATDFs also using bit-by-bit encryption, but where the underlying one-bit encryption scheme is not CCA-secure - namely, we use the "naïve" one-bit scheme described above. We show this one-bit scheme is uniquely RR and moreover satisfies a notion of DCCA with analogous "enhanced" security (where the decryption oracle also returns coins).

More Efficient Schemes. We note that the above is a feasibility result in terms of minimal assumptions. We also show more efficient constructions of ECCAsecure encryption from tag-based ATDFs as defined in [25] and from ATDFs having a large number of simultaneous hardcore bits (using the KEM/DEM paradigm). See Section 4.2 and the full version [14].

\subsection{Applications to Adaptive Trapdoor Functions}

Going the other direction, we next give applications of ECCA-security to the theory of adaptive TDFs. Namely, we show (1) adaptive TDFs are in fact equivalent to uniquely randomness-recovering ECCA-secure PKE. This helps us better understand the power and complexity of ATDFs. We furthermore show (2) "tagbased" ATDFs as defined in [25] are likewise equivalent to uniquely randomnessrecovering ECCA-secure PKE. A corollary of (1) and (2) is that tag-based and non-tag-based ATDFs are themselves equivalent, which resolves a foundational question left open by 25. We note that it is in fact much easier to construct uniquely RR ECCA-secure PKE from tag-based ATDFs than from non-tagbased ATDFs. (The rough intuition is that in the tag-based case, a signature scheme can be used to "glue together" many one-bit encryptions via a common tag, namely a single verification key.) Indeed, the apparent extra power 
of tag-based ATDFs makes it surprising that they turn out to be equivalent to (non-tag-based) ATDFs. We note that unlike the TDF case, the equivalence of tag-based and standard PKE is much easier to prove [26].

\subsection{Applications to PKE with Non-interactive Opening}

PKENO. Public-key encryption with non-interactive opening (PKENO), introduced by Damgård an Thorbeck 16 and studied in detail by 151920, allows a receiver to non-interactively prove to anyone that a ciphertext $c$ decrypts to a message $m$. As discussed in the above-mentioned work, PKENO has applications to multiparty computation (e.g., auctions and elections), secure message transmission, group signatures, and more. But despite numerous applications, such schemes have been difficult to realize. Secure constructions of PKENO currently exist from identity-based encryption [15] and robust non-interactive threshold encryption [20], which are somewhat heavy-weight primitives.

Resurrecting a Simple Approach. We show that ECCA-secure encryption can be used to securely realize (for the first time) a simple approach to PKENO originally suggested by [16. The basic idea is to use a randomness-recovering PKE and have the receiver provide the recovered coins as the proof. However, several issues need to be addressed for this approach to work. One problem already discussed in [20, Sect. 4.1] is that there must be a way for the receiver to prove the claimed behavior of the decryption algorithm on ciphertexts that are not an output of the encryption algorithm, and for which necessarily no underlying coins exist. (Note that such ciphertexts may or may not decrypt to $\perp$ in general.) More fundamentally, we observe that the encryption scheme must be ECCA secure (which was not even defined in prior work); standard chosen-ciphertext security is not enough, because here the adversary in the corresponding PKENO security game has the ability to see random coins underlying ciphertexts of its choosing. We now describe our results in more detail.

PKENO-Compatible ECCA Encryption. First, we formalize a notion of PKENO-compatible ECCA-secure encryption, for which we can overcome the above problems and safely use the underlying message and randomness as the non-interactive opening of a ciphertext. There are two requirements for such a scheme: (1) It has a "partial-randomness" recovery algorithm that, informally, recovers enough coins to uniquely identify the underlying message. (Here "full" randomness-recovery is not needed, and would not permit constructions where the ciphertext contains randomized parts that are verifiable without coins, like a one-time signature or zero-knowledge proof.) This should also be true for ciphertexts outside the range of the encryption algorithm but which do not decrypt to $\perp 2$

${ }^{2}$ For example, consider a scheme that always outputs ciphertexts whose last bit is "0," but whose decryption algorithm ignores this last bit. Then clearly we can still recover the randomness underlying ciphertexts ending in " 1 " despite the fact that such ciphertexts are outside the range of the encryption algorithm. 
(2) The scheme has ciphertext verifiability, meaning one can check without the secret key (but possibly with the help of the recovered partial coins) whether the decryption of a ciphertext is $\perp$. We define ECCA security of such schemes with respect to the partial-randomness recovery algorithm.

We also define an analogous notion of PKENO-compatible ECCA-secure tagbased PKE. We show that one can efficiently transform such a scheme into a (non-tag-based) PKENO-compatible ECCA-secure PKE scheme using either of the two "BCHK transforms" 9]. (Recall that [9] give a "basic" transform using one-time signatures and a "more efficient" transform based on symmetric-key primitives.)

Constructing PKENO. We show a generic way to achieve PKENOcompatibility from any ECCA-secure RR PKE by adding a non-interactive zeroknowledge (NIZK) proof of "well-formedness" to a ciphertext, namely that there exist some underlying message and random coins. (The idea of adding such a proof to achieve PKENO comes from [1620, although not in connection with ECCA.) For this approach to work, the PKE scheme does not need to be uniquely RR, but it needs perfect correctness. Moreover, we show the NIZK needs to be simulation-sound.

While this construction is generic, it is also inefficient. Towards more efficient schemes, we show our construction of ECCA-secure tag-based PKE from tagbased ATDFs can be made PKENO-compatible if its starting tag-based ATDF has "range verifiability", meaning that anyone can verify preimage existence of a range point. We propose two efficient such tag-based ATDFs. The first instantiates a general tag-based ATDF construction from [25] using a lossy and all-butone TDF as defined in 29. Specifically, we use the lossy and all-but-one TDFs of Freeman et al. [18] based on the decision-linear (DLIN) assumption. We show that in this case preimage existence is a "Groth-Sahai" statement 22], for which we know efficient NIZK constructions in bilinear groups 3 Interestingly, we show simulation-soundness is not needed in this case, illustrating another efficiency benefit over the generic approach. The second is a tag-based ATDF from 25] based on the "instance-independent" RSA assumption, which we observe intrinsically has range verifiability because it is a permutation. The resulting PKENO scheme based on II-RSA is quite practical.

\subsection{Related Work}

ECCA is similar in spirit to coin-revealing selective opening attack (SOAC) $[101738$. In the latter setting, there are say $n$ ciphertexts encrypting related (but unknown) messages under independent random coins, and the adversary requests the plaintexts and random coins corresponding to some subset of them; the question is whether the "unopened" ciphertexts remain secure. However, it

\footnotetext{
3 Technically, when the NIZK is added, the tag-based ATDF is not a trapdoor function anymore but is already a tag-based PKE scheme (because the NIZK part is randomized), but we gloss over this technicality in our informal exposition.
} 


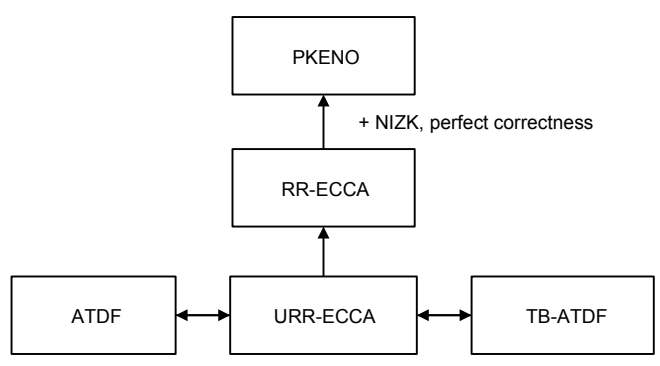

Fig. 1. Relations between various primitives studied in this paper. "(U)RR-ECCA" is (uniquely) randomness-recovering enhanced-chosen-ciphertext secure PKE, "(TB-) ATDF" is (tag-based) adaptive trapdoor function, and "PKENO" is public-key encryption with non-interactive opening.

seems to us that SOA-C is neither implied by, nor implies, ECCA. It is an interesting question whether ECCA has any applications in the domain of SOA-C.

An analogue of ECCA (in the case of inefficient randomness-recovery) has been previously defined for commitment schemes by Canetti et al. [12, which they call CCA-secure commitments. These are commitment schemes that remain secure when the adversary has access to an unbounded decommitment oracle that it can call on commitments other than the challenge. They are interested in such schemes that are interactive but in the plain model, meaning there are no public keys. Thus, our setting seems incomparable (as we disallow interaction but allow public keys). However, we view their work as supporting the claim that ECCA is a natural notion of security to consider for encryption.

Other variants of CCA-security for encryption considered before include replayable CCA security [11, constrained CCA security [23, and detectable CCA security 24]. Notably, these are all relaxations of CCA security, whereas we consider a strengthening. Another strengthening of CCA security previously considered is plaintext awareness [7/25].

\section{Preliminaries}

\subsection{Notation and Conventions}

If $A$ is an algorithm then $y \leftarrow A\left(x_{1}, \ldots, x_{n} ; r\right)$ means we run $A$ on inputs $x_{1}, \ldots, x_{n}$ and coins $r$ and denote the output by $y$. By $y \leftarrow \$ A\left(x_{1}, \ldots, x_{n}\right)$ we denote the operation of picking $r$ at random and letting $y \leftarrow A\left(x_{1}, \ldots, x_{n} ; r\right)$. Unless otherwise indicated, an algorithm may be randomized. The security parameter is denoted $k \in \mathbb{N}$. We say that an algorithm is efficient if it is probabilistic polynomial time in the security parameter. All algorithms we consider are efficient unless indicated otherwise. 


\subsection{Public-Key Encryption}

A public-key encryption scheme [21] with message space $M s g S p$ is a triple of algorithms PKE $=(\mathrm{Kg}$, Enc, Dec). The key-generation algorithm Kg returns a public key $p k$ and matching secret key sk. The encryption algorithm Enc takes $p k$ and a plaintext $m$ to return a ciphertext. The deterministic decryption algorithm Dec takes sk and a ciphertext $c$ to return a plaintext.

Correctness. An issue that will be more important than usual in our context is correctness, which refers to how likely it is that an encrypted message decrypts to some other message. By default we require perfect correctness: for all $k \in \mathbb{N}$ and $m \in \operatorname{MsgSp}\left(1^{k}\right)$,

$$
\operatorname{Pr}\left[\operatorname{Dec}(s k, \operatorname{Enc}(p k, m))=m:(p k, s k) \leftarrow s \operatorname{Kg}\left(1^{k}\right)\right]=1 .
$$

If instead we allow this probability to be $1-\nu(k)$ we say that that PKE has decryption error $\nu(\cdot)$.

Tag-Based. PKE is tag-based [26 with tag space TagSp if Enc and Dec take an additional input $t \in \operatorname{TagSp}\left(1^{k}\right)$ called the $\operatorname{tag}$. Again, by default we require perfect correctness: for all $k \in \mathbb{N}, m \in \operatorname{MsgSp}\left(1^{k}\right)$, and $t \in \operatorname{TagSp}\left(1^{k}\right)$, $\operatorname{Pr}\left[\operatorname{Dec}(s k, t, \operatorname{Enc}(p k, t, m))=m:(p k, s k) \leftarrow s \operatorname{Kg}\left(1^{k}\right)\right]=1$. Decryption error is defined analogously.

Other Standard Primitives. We recall the definitions of other standard primitives such as (injective) trapdoor functions in the full version [14.

\section{Enhanced Chosen-Ciphertext Security}

Randomness Recovery. We start with a definition of randomness recovery for public-key encryption. For any public-key encryption scheme PKE $=(\mathrm{Kg}, \mathrm{Enc}, \mathrm{Dec})$ we specify an additional randomness-recovery algorithm Rec that takes a secret key sk and ciphertext $c$ to return coins $r$. To our knowledge, this notion has been discussed informally in the literature (e.g. in [29]) but our formalization is novel. Suppose Enc draws its coins from Coins. We require that for all messages $m \in$ $\operatorname{MsgSp}\left(1^{k}\right)$,

$$
\begin{aligned}
\operatorname{Pr}\left[\operatorname{Enc}\left(p k, m ; r^{\prime}\right) \neq c:(p k, s k) \leftarrow \$ \mathrm{Kg} ;\right. & r \leftarrow{ }^{\operatorname{Coins}\left(1^{k}\right) ;} \\
& \left.c \leftarrow \operatorname{Enc}(p k, m ; r) ; r^{\prime} \leftarrow \operatorname{Rec}(s k, c)\right]
\end{aligned}
$$

is negligible. Note that we do not necessarily require $r=r^{\prime}$; that is, the randomness recovery algorithm need not return the same coins used for encryption; indeed, it may not be possible, information theoretically, to determine $r$ from sk and $c$. We also do not require Rec to be efficient in general. But in the special case that Rec is polynomial-time we say that PKE is randomness recovering (RR). Moreover, if the forgoing condition on Rec holds for $r^{\prime}=r$ we say that 
PKE is uniquely randomness recovering In the definition that follows these are important special cases, but they are not assumed by the definition.

In the tag-based case, Rec also takes a $t a g$ as input and we require that for all $m \in \operatorname{MsgSp}\left(1^{k}\right)$ and $t \in \operatorname{TagSp}\left(1^{k}\right)$, the following is negligible:

$\operatorname{Pr}\left[\operatorname{Enc}\left(p k, t, m ; r^{\prime}\right) \neq c:(p k, s k) \leftarrow \$ \mathrm{Kg} ; r \leftarrow \$ \operatorname{Coins}\left(1^{k}\right) ; c \leftarrow \operatorname{Enc}(p k, t\right.$, $\left.m ; r) ; r^{\prime} \leftarrow \operatorname{Rec}(s k, t, c)\right]$ is negligible. Randomness-recovery and unique RR are defined analogously.

ECCA Definition. We are now ready to state our new definition. Let PKE = $(\mathrm{Kg}$, Enc, Dec) be a public-key encryption scheme. We associate to PKE and an adversary $A=\left(A_{1}, A_{2}\right)$ an enhanced chosen-ciphertext attack experiment:

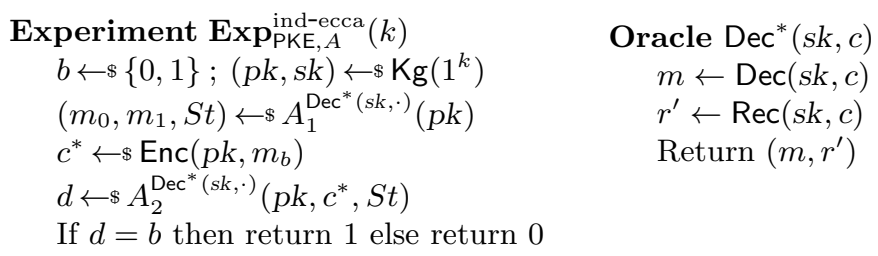

Above we require that the output of $A_{1}$ satisfies $\left|m_{0}\right|=\left|m_{1}\right|$ and that $A_{2}$ does not query $c^{*}$ to its oracle. Define the ind-ecca advantage of $A$ against PKE as

$$
\operatorname{Adv}_{\mathrm{PKE}, A}^{\text {ind-ecca }}(k)=2 \cdot \operatorname{Pr}\left[\operatorname{Exp}_{\mathrm{PKE}, A}^{\text {ind-ecca }}(k) \text { outputs } 1\right]-1 .
$$

We say that PKE is enhanced chosen-ciphertext secure (ECCA-secure) if $\operatorname{Adv}_{\mathrm{PKE}, A}^{\text {ind-ecca }}(\cdot)$ is negligible for every efficient $A$.

Note that when PKE is randomness recovering, the ECCA experiment is efficient. In general, however, one can still ask whether a scheme meets the notion of ECCA even when it is not RR. In this case, it may still be possible to simulate the ECCA experiment efficiently since in the proof of security we are additionally given the code of the adversary $A$ (and so, for example, the randomness for encryption might be efficiently extractable from the code of $A$ using non-blackbox techniques). We leave exploration of ECCA security relative to an inefficient Rec algorithm for future work.

(Not) Allowing Decryption Error. Unless otherwise specified, we will always require that an ECCA-secure PKE scheme has perfect correctness. Indeed, in the full version [14] we show how to construct an ECCA-secure, randomnessrecovering $\mathrm{PKE}$ scheme given any $\mathrm{CCA}$-secure one if we allow negligible decryption error - however, an ECCA-secure scheme with negligible decryption error will not be sufficient in the applications we consider 5 This observation and example are due to [1].

\footnotetext{
${ }^{4}$ Looking ahead, it turns out that in some applications of ECCA, non-unique randomness recovery is $\mathrm{OK}$ as long as the scheme has perfect correctness.

5 The resulting ECCA-secure scheme does not have unique randomness recovery, though. In the case of unique randomness recovery, schemes with negligible decryption error may still have some applications, but for simplicity we do not discuss it in the paper.
} 
CCA Does Not Imply ECCA. A next natural question to ask is whether, assuming perfect correctness, ECCA security is a stronger requirement than CCA security. We answer this question affirmatively by showing that, given a perfectly correct, CCA-secure randomness-recovering PKE scheme, we can construct another RR PKE scheme that is still CCA-secure but is not ECCA-secure. This motivates the construction of specialized ECCA-secure schemes in Section 4 .

Consider a RR CCA-secure scheme PKE $=(\mathrm{Kg}$, Enc, Dec). We transform PKE to a new scheme PKE* $=\left(\mathrm{Kg}^{*}, \mathrm{Enc}^{*}, \mathrm{Dec}^{*}\right)$ which is still CCA-secure but is not ECCA-secure. The idea is to embed a "test" ciphertext in the public key of the new scheme, such that its decryption algorithm returns the secret key if given as input some randomness consistent with this test ciphertext. Formally, PKE* is constructed as follows (where we implicitly assume the public key is contained in the secret key):

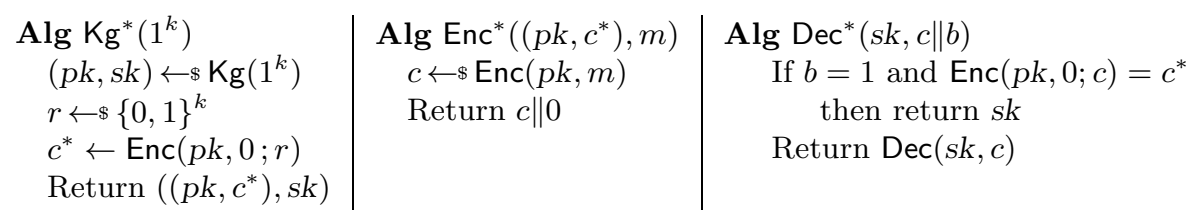

Note that using the extra "flag bit" appended to ciphertexts ensures that PKE* maintains perfect correctness. We prove the following proposition in the full version [14.

Proposition 1. Assuming PKE is CCA-secure and has perfect correctness, $\mathrm{PKE}^{*}$ is $C C A$-secure but is not ECCA-secure.

Tag-Based Definition. Let TB-PKE $=(\mathrm{Kg}$, Enc, Dec) be a tag-based publickey encryption scheme with tag space TagSp. We associate to TB-PKE and an adversary $A=\left(A_{1}, A_{2}, A_{3}\right)$ a tag-based enhanced chosen-ciphertext attack experiment,

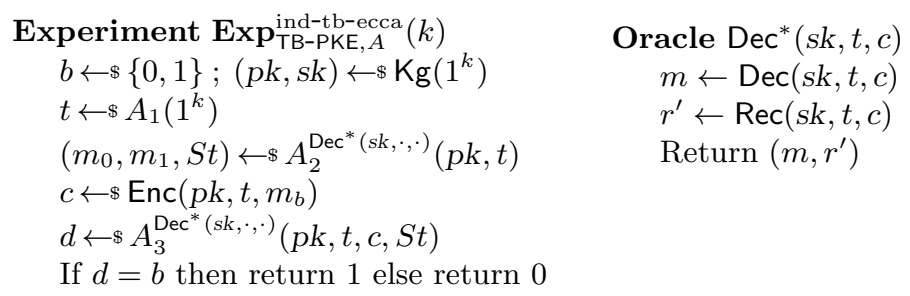

Above we require that the output of $A_{2}$ satisfies $\left|m_{0}\right|=\left|m_{1}\right|$ and that $A_{3}$ does not make a query of the form $\operatorname{Dec}^{*}(s k, t, \cdot)$ to its oracle. Define the ind-tb-ecca advantage of $A$ against PKE as

$$
\operatorname{Adv}_{\mathrm{PKE}, A}^{\text {ind-tb-ecca }}(k)=2 \cdot \operatorname{Pr}\left[\operatorname{Exp}_{\mathrm{PKE}, A}^{\text {ind-tb-ecca }}(k) \text { outputs } 1\right]-1 .
$$

We say that TB-PKE is tag-based enhanced chosen-ciphertext secure (TB-ECCAsecure) if $\mathbf{A d v}_{\mathrm{PKE}, A}^{i n d-t b-e c c a}(\cdot)$ is negligible for every efficient $A$. 


\section{Constructions of ECCA-Secure PKE}

We give several constructions of ECCA secure encryption, which are based on notions of adaptivity for trapdoor functions introduced in 25] (see the full version [14]).

\subsection{ECCA Security from Adaptive Trapdoor Functions}

Here we construct ECCA-secure public-key encryption from adaptive TDFs. We note that our construction applies to general ATDFs; in the case of ATDFs with a linear number of hardcore bits we obtain a much more efficient construction; see the full version 14 for details.

Overview and Intuition. As in 25] (which constructs CCA-secure PKE from ATDFs), our approach involves first constructing a one-bit encryption scheme and then transforming it into a multi-bit scheme. In doing so we heavily use the recent approach of Hohenberger et al. 24] and their notion of detectable CCA security (DCCA); this should be contrasted with [25] who rely on 27] instead. Let us explain why.

Both [24] and 27] provide a way to "tie together" many one-bit ciphertexts via "inner" and "outer" encryption layers but differ in which layer contains the onebit ciphertexts. In [27, the inner layer is a multi-bit $q$-bounded non-malleable encryption scheme while the outer layer is the concatenation of one-bit ciphertexts. This means that without a randomness-recovering inner layer, 27] does not preserve randomness-recovery of the outer one-bit scheme. Such an inner layer seems hard to construct, as known approaches to non-malleability [28113] crucially use randomness in an un-invertible way in their encryption algorithms (e.g., to generate a signature key-pair or a zero-knowledge proof).

On the other hand, in Hohenberger et al. 24] it is the inner layer that is the concatenation of one-bit ciphertexts, which obviates the problem since this inner layer is also used to encrypt randomness for use by the outer layer and thus the latter does not need to be randomness-recovering for the overall scheme to be so. Surprisingly, we also show that when this inner layer is RR then in all hybrid games used for the security proof the simulator is even able to the return randomness corresponding to valid ciphertexts, and thus the overall scheme also has ECCA security.

Enhanced DCCA Security. The notion of detectable chosen-ciphertext (DCCA) security was recently introduced by 24. We define here the notion of enhanced DCCA (EDCCA) security, which parallels the notion of enhanced CCA security. In our definition, we require that the DCCA scheme be both enhanced and RR, because our application of DCCA requires both properties.

A detectable encryption scheme consists of a public-key encryption scheme $\left(\mathrm{Kg}\right.$, Enc, Dec) and a detecting function $\mathcal{F}:\left(p k, c^{\prime}, c\right) \mapsto b \in\{0,1\}$ mapping a public key and two ciphertexts to a bit. The detecting function must satisfy unpredictability, which informally means that given the description of $\mathcal{F}$ and a public key $p k$, it should be hard to find a ciphertext $c^{\prime}$ that is related to a 
"challenge" ciphertext $c$, in that $\mathcal{F}\left(p k, c^{\prime}, c\right)=1$, before being given $c$. See 24 for the formal definition.

Definition. We define enhanced detectable chosen ciphertext security for a RR scheme $\mathrm{PKE}=(\mathrm{Kg}, \mathrm{Enc}, \mathrm{Dec}, \mathrm{Rec})$ and an unpredictable detecting function $\mathcal{F}$ similarly to ECCA in Section 3, except that the decryption oracle for $A_{2}$ returns $\perp$ whenever it is queried on a ciphertext $c$ such that $\mathcal{F}\left(p k, c^{*}, c\right)=1$, where $c^{*}$ is the challenge ciphertext. (see [14]).

EDCCA Security from ATDFs. Let TDF $=(T d g$, Eval, Inv $)$ be a trapdoor function with hardcore bit hc. We define the following multi-bit PKE scheme $\mathrm{EDCCA}[\mathrm{TDF}]=\left(\mathrm{Kg}_{\mathrm{D}}, \mathrm{Enc}_{\mathrm{D}}, \mathrm{Dec}_{\mathrm{D}}\right)$ with message space $\{0,1\}^{\ell}$ :

$$
\begin{array}{c|c|c}
\operatorname{Alg} \operatorname{Kg}_{\mathrm{D}}\left(1^{k}\right) & \operatorname{Alg} \operatorname{Enc}_{\mathrm{D}}\left(e k, m=m_{1}, \ldots, m_{\ell}\right) & \operatorname{Alg} \operatorname{Dec}(t d, C) \\
(e k, t d) & x_{1} \leftarrow \$\{0,1\}^{k} ; \ldots ; x_{\ell} \leftarrow \$\{0,1\}^{k} & \operatorname{Parse} C=\left(y_{1}, \beta_{1}, \ldots, y_{\ell}, \beta_{\ell}\right) \\
\leftarrow \$ \operatorname{Tdg}\left(1^{k}\right) & \operatorname{Return} C=\left(\operatorname{Eval}\left(e k, x_{1}\right),\right. & \text { For } 1 \leq i \leq \ell \\
\operatorname{Return}(e k, t d) & \operatorname{hc}\left(x_{1}\right) \oplus m_{1}, \ldots, & m_{i}=\mathrm{hc}\left(\operatorname{Inv}\left(t d, y_{i}\right)\right) \oplus \beta_{i} \\
& \left.\operatorname{Eval}\left(e k, x_{\ell}\right), \operatorname{hc}\left(x_{\ell}\right) \oplus m_{\ell}\right) & \operatorname{Return~} m_{1}, \ldots, m_{\ell}
\end{array}
$$

In [14] we show that if TDF is adaptive one-way then this scheme is EDCCA with respect to the detection function $\mathcal{F}_{\mathrm{D}}$, which on input pk, $C^{*}=$ $\left(y_{1}^{*}, \beta_{1}^{*}, \ldots, y_{\ell}^{*}, \beta_{\ell}^{*}\right)$ and $C=\left(y_{1}, \beta_{1}, \ldots, y_{\ell}, \beta_{\ell}\right)$ outputs 1 iff for some $i, j \in[\ell]$ : $y_{i}^{*}=y_{j}$.

Scheme EDCCA[TDF] is perfectly correct and uniquely RR, which will be crucial for our application to ATDFs in Section 5. We also wish to stress that it gives a novel example of a DCCA secure scheme; our scheme is not the concatenation of ciphertexts for a 1-bit CCA-secure scheme. Indeed, a ciphertext of the form (Eval $(e k, x), \mathrm{hc}(x) \oplus m)$ is trivially malleable by flipping the second component.

From EDCCA to ECCA Security. We next show that the construction of a CCA-secure scheme from a DCCA-secure one in 24] allows us to go from EDCCA to ECCA. That is, we show that the construction preserves "enhanced" security; it also preserves (unique) RR. Specifically, we instantiate the construction of [24] with the following components:

$\Pi_{\mathrm{D}}$, the above RR EDCCA scheme EDCCA[TDF];

$\Pi_{\mathrm{CPA}}$, a CPA-secure scheme with perfect correctness (which can also be instantiated with EDCCA[TDF]); and

$\Pi_{1 \mathrm{~b}}$, a perfectly correct 1 -bounded CCA-secure6 (which can be constructed from a multi-bit CPA scheme via the construction from [13]).

Note that all these components can be constructed in a black-box manner from ATDFs. In the full version [14] we prove that the following is a multi-bit encryption scheme with message space $\{0,1\}^{\ell}$ that is uniquely RR, ECCA-secure and perfectly correct.

${ }^{6}$ 1-bounded CCA security means that the adversary may make only a single decryption query. 


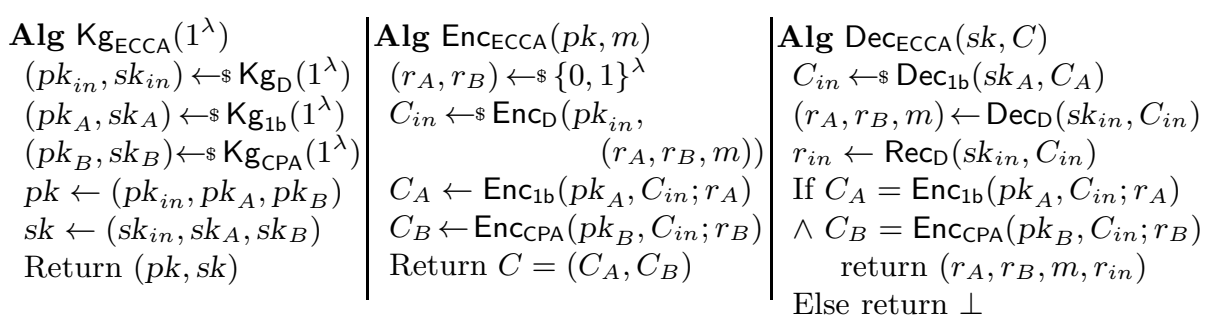

\subsection{ECCA Security from Tag-Based ATDFs}

We give more efficient constructions of ECCA-secure public-key encryption from tag-based adaptive trapdoor functions, introduced by Kiltz et al. [25]. Due to space constraints, these constructions are deferred to [14].

\section{Application to Adaptive Trapdoor Functions}

We use ECCA-security as a unifying concept to show that the notions of adaptive TDFs and tag-based adaptive ATDFs introduced by Kiltz et al. 25] are equivalent (via fully black-box reductions), resolving a foundational open question raised in 25. To do so, we show that both primitives are implied by uniquely randomness-recovering ECCA-secure PKE. Combined with Section 4 , this shows that in fact uniquely RR PKE, adaptive TDFs, and tag-based ATDFs are all equivalent. Due to space constraints, these implications are deferred to the full version [14.

\section{Application to PKE with Non-interactive Opening}

In this section, we show that ECCA-secure encryption is a natural building block for public key encryption with non-interactive opening (PKENO) [16]15]19]20. PKENO allows the receiver to non-interactively prove that a given ciphertext decrypts to a claimed message. Our constructions yield new and practical PKENO schemes.

PKENO extends a public-key encryption scheme PKE $=(\mathrm{Kg}$, Enc, Dec) by the following algorithms: Prove takes a secret key sk and a ciphertext $c$, and outputs a proof $\pi$. Ver takes a public key $p k$, a ciphertext $c$, a plaintext $m$ and a proof $\pi$, and outputs 0 or 1 . We require proof correctness: for all ciphertexts (i.e. strings) $c, \operatorname{Pr}\left[\operatorname{Ver}(p k, c, \operatorname{Dec}(s k, c), \operatorname{Prove}(s k, c)) \neq 1:(p k, s k) \leftarrow s \operatorname{Kg}\left(1^{k}\right)\right]$ is negligible.

Security. In [15]19 security of PKENO is defiend by indistinguishability under chosen-ciphertext and -proof attacks (IND-CCPA) and proof soundness. The former guarantees that a ciphertext hides the plaintext even when the adversary sees decryptions of and proofs for other ciphertexts; the latter formalizes that no adversary should be able to produce a proof for a message and ciphertext that is not the encryption of that message. 


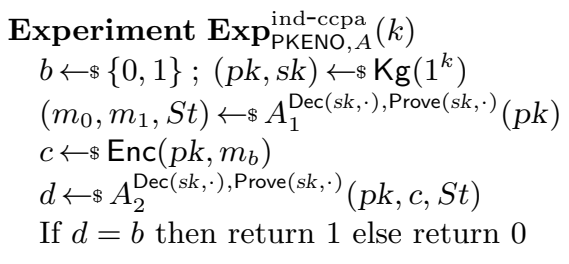
Experiment $\operatorname{Exp}_{\mathrm{PKENO}, A}^{\text {proof }},(k)$
$(p k, s k) \leftarrow \$ \operatorname{Kg}\left(1^{k}\right)$
$\left(m^{\prime}, \pi^{\prime}, c^{\prime}\right) \leftarrow \$ A(p k, s k)$
$m \leftarrow \operatorname{Dec}\left(s k, c^{\prime}\right)$
If $\operatorname{Ver}\left(p k, c^{\prime}, m^{\prime}, \pi^{\prime}\right)=1$ and $m \neq m^{\prime}$
then return 1 ; else return 0

Fig. 2. Security experiments for PKENO

Formally, to a scheme PKENO $=(\mathrm{Kg}$, Enc, Dec, Prove, Ver $)$, and an adversary $A=\left(A_{1}, A_{2}\right)$ we associate the chosen-ciphertext and -proof attack experiment given in Figure 2. We require that the output of $A_{1}$ satisfies $\left|m_{0}\right|=\left|m_{1}\right|$ and that $A_{2}$ does not query $c$ to any of its oracles. We say that PKENO is chosen-ciphertext and-proof-attack secure (CCPA-secure) if $2 \cdot \operatorname{Pr}\left[\operatorname{Exp}_{\mathrm{PKENO}, A}^{\text {ind-ccpa }}(k)\right.$ outputs 1$]-1$ is negligible for every efficient $A$. We associate to a scheme PKENO and an adversary $A=\left(A_{1}, A_{2}\right)$ a proof-soundness experiment, given in Figure 2] and say that PKENO is proof-sound if $\operatorname{Pr}\left[\operatorname{Exp}_{\mathrm{PKENO}, A}^{\text {proof-snd }}(k)\right.$ outputs 1$]$ is negligible for every efficient $A$.

We note that in contrast to [1519] our definition of proof soundness also considers adversarially produced ciphertexts, which need not even be a valid output of the encryption algorithm. Note that it is already required by proof correctness that the PKENO correctly proves decryption of such ciphertexts (which may or may not decrypt to $\perp$ ), so constructions should achieve this stronger notion of proof soundness anyway.

Strong Proof Soundness. An even stronger notion of proof soundness is defined in 20, which also handles maliciously chosen receiver public keys. In the full version [14] we define notions of strong proof soundness and discuss how our constructions can be adapted to meet them.

\subsection{PKENO-Compatible ECCA-Secure PKE}

A natural approach to building PKENO suggested by [16] is to use a randomnessrecovering encryption scheme and have the receiver provide the recovered coins as the proof. A moment's reflection reveals that for this approach to work, the encryption scheme must be ECCA secure in order to protect against chosenproof attacks. In addition, as discussed in [161520], we also need a way for the receiver to prove correct decryption of ciphertexts that are not in the range of the encryption algorithm, in which case such coins may not be defined. In this section we define a notion of PKENO-compatible ECCA-secure encryption for which we can do this. Below we discuss the properties such a scheme must have, but due to space constraints, we defer the details to the full version [14].

Partial-Randomness Recovery. It turns out that for such schemes we do not always achieve, nor need, the notion of full RR, so we define a natural generalization we call partial-randomness recovery, which loosely says that enough 
of the random coins are recovered to uniquely identify the underlying message. However, in order to deal with the case that ciphertexts outside the range of the encryption algorithm may not decrypt to $\perp$, we also strengthen what we get from RR encryption in some respect.

Ciphertext Verifiability. This notion intuitively means a verifier can check (with the help of some partial random coins) whether the decryption algorithm returns $\perp$ on a given ciphertext.

PKENO-Compatibility. We say PKE is a PKENO-compatible ECCA-secure $P K E$ scheme if it satisfies ECCA-security, partial-randomness recovery and ciphertext verifiability. In the full version of the paper [14] we show that a PKENOcompatible ECCA-secure PKE scheme indeed gives us PKENO by using the idea of [16] described above. We also show an analogous theorem in the case of tagbased PKE.

\subsection{PKENO-Compatible PKE Using NIZK}

PKENO-compatibility can be obtained generically from any ECCA-secure RR PKE by adding a non-interactive zero-knowledge proof (NIZK) of ciphertext "well-formedness." The approach of using a NIZK originates from [1520], although not with respect to ECCA-secure encryption. We note that we do not require the starting ECCA-secure encryption scheme to be uniquely RR (although our constructions in Section 4 achieve this), but it should have perfect correctness. Moreover, the NIZK needs to be simulation-sound, for reasons analogous to the proof of full anonymity of the group signature construction in [4]. See [14] for the details.

\subsection{Efficient PKENO-Compatible Tag-Based PKE}

Our construction using NIZKs, while it applies to any ECCA-secure RR PKE scheme, is not very efficient unless we rely on the random-oracle model [6] for the NIZK. We show more efficient constructions by following the tag-based approach; namely, we show that our construction from tag-based ATDFs in Section 4.2 can be made PKENO-compatible by using special tag-based ATDFs (from which we can then obtain non-tag-based PKENO-compatible PKE). The idea is to use tag-based ATDF for which we have "range verifiability," meaning that anyone can verify preimage existence. In our first construction, we achieve this property by adding an efficient NIZK proof due to Groth and Sahai [22. In our second construction, we use a tag-based ATDF that has this property because it is a permutation. Details of these constructions are again deferred to [14].

Acknowledgements. We gratefully acknowledge Mihir Bellare, whose comments improved our results and presentation. The second author was supported by EPSRC grant EP/H043454/1 and the European Research Council, ERC Starting Grant (259668-PSPC). The fourth author was supported by NSF grants CNS-1012910 and CNS-0546614. 


\section{References}

1. Bellare, M.: Private communication (2012)

2. Bellare, M., Desai, A., Pointcheval, D., Rogaway, P.: Relations among notions of security for public-key encryption schemes. In: Krawczyk, H. (ed.) CRYPTO 1998. LNCS, vol. 1462, pp. 26-45. Springer, Heidelberg (1998)

3. Bellare, M., Hofheinz, D., Yilek, S.: Possibility and impossibility results for encryption and commitment secure under selective opening. In: Joux, A. (ed.) EUROCRYPT 2009. LNCS, vol. 5479, pp. 1-35. Springer, Heidelberg (2009)

4. Bellare, M., Micciancio, D., Warinschi, B.: Foundations of group signatures: Formal definitions, simplified requirements, and a construction based on general assumptions. In: Biham, E. (ed.) EUROCRYPT 2003. LNCS, vol. 2656, pp. 614-629. Springer, Heidelberg (2003)

5. Bellare, M., Palacio, A.: Towards plaintext-aware public-key encryption without random oracles. In: Lee, P.J. (ed.) ASIACRYPT 2004. LNCS, vol. 3329, pp. 48-62. Springer, Heidelberg (2004)

6. Bellare, M., Rogaway, P.: Random oracles are practical: A paradigm for designing efficient protocols. In: Ashby, V. (ed.) ACM CCS 1993, pp. 62-73. ACM Press (November 1993)

7. Bellare, M., Rogaway, P.: Optimal asymmetric encryption. In: De Santis, A. (ed.) EUROCRYPT 1994. LNCS, vol. 950, pp. 92-111. Springer, Heidelberg (1995)

8. Bellare, M., Yilek, S.: Encryption schemes secure under selective opening attack. Cryptology ePrint Archive, Report 2009/101 (2009), http://eprint.iacr.org/

9. Boneh, D., Canetti, R., Halevi, S., Katz, J.: Chosen-ciphertext security from identity-based encryption. SIAM Journal on Computing 36(5), 1301-1328 (2007)

10. Canetti, R., Feige, U., Goldreich, O., Naor, M.: Adaptively secure multi-party computation. In: 28th ACM STOC, pp. 639-648. ACM Press (May 1996)

11. Canetti, R., Krawczyk, H., Nielsen, J.B.: Relaxing chosen-ciphertext security. In: Boneh, D. (ed.) CRYPTO 2003. LNCS, vol. 2729, pp. 565-582. Springer, Heidelberg (2003)

12. Canetti, R., Lin, H., Pass, R.: Adaptive hardness and composable security in the plain model from standard assumptions. In: 51st FOCS, pp. 541-550. IEEE Computer Society Press (October 2010)

13. Choi, S.G., Dachman-Soled, D., Malkin, T., Wee, H.: Black-box construction of a non-malleable encryption scheme from any semantically secure one. In: Canetti, R. (ed.) TCC 2008. LNCS, vol. 4948, pp. 427-444. Springer, Heidelberg (2008)

14. Dachman-Soled, D., Fuchsbauer, G., Mohassel, P., O'Neill, A.: Enhanced chosenciphertext security and applications. Cryptology ePrint Archive, Report 2012/543 (2012)

15. Damgård, I., Hofheinz, D., Kiltz, E., Thorbek, R.: Public-key encryption with non-interactive opening. In: Malkin, T. (ed.) CT-RSA 2008. LNCS, vol. 4964, pp. 239-255. Springer, Heidelberg (2008)

16. Damgård, I.B., Thorbek, R.: Non-interactive proofs for integer multiplication. In: Naor, M. (ed.) EUROCRYPT 2007. LNCS, vol. 4515, pp. 412-429. Springer, Heidelberg (2007)

17. Dwork, C., Naor, M., Reingold, O., Stockmeyer, L.J.: Magic functions. Journal of the ACM 50(6), 852-921 (2003)

18. Freeman, D.M., Goldreich, O., Kiltz, E., Rosen, A., Segev, G.: More constructions of lossy and correlation-secure trapdoor functions. In: Nguyen, P.Q., Pointcheval, D. (eds.) PKC 2010. LNCS, vol. 6056, pp. 279-295. Springer, Heidelberg (2010) 
19. Galindo, D.: Breaking and repairing damgård et al. public key encryption scheme with non-interactive opening. In: Fischlin, M. (ed.) CT-RSA 2009. LNCS, vol. 5473, pp. 389-398. Springer, Heidelberg (2009)

20. Galindo, D., Libert, B., Fischlin, M., Fuchsbauer, G., Lehmann, A., Manulis, M., Schröder, D.: Public-key encryption with non-interactive opening: New constructions and stronger definitions. In: Bernstein, D.J., Lange, T. (eds.) AFRICACRYPT 2010. LNCS, vol. 6055, pp. 333-350. Springer, Heidelberg (2010)

21. Goldwasser, S., Micali, S.: Probabilistic encryption. Journal of Computer and System Sciences 28(2), 270-299 (1984)

22. Groth, J., Sahai, A.: Efficient non-interactive proof systems for bilinear groups. In: Smart, N.P. (ed.) EUROCRYPT 2008. LNCS, vol. 4965, pp. 415-432. Springer, Heidelberg (2008)

23. Hofheinz, D., Kiltz, E.: Secure hybrid encryption from weakened key encapsulation. In: Menezes, A. (ed.) CRYPTO 2007. LNCS, vol. 4622, pp. 553-571. Springer, Heidelberg (2007)

24. Hohenberger, S., Lewko, A., Waters, B.: Detecting dangerous queries: A new approach for chosen ciphertext security. In: Pointcheval, D., Johansson, T. (eds.) EUROCRYPT 2012. LNCS, vol. 7237, pp. 663-681. Springer, Heidelberg (2012)

25. Kiltz, E., Mohassel, P., O'Neill, A.: Adaptive trapdoor functions and chosenciphertext security. In: Gilbert, H. (ed.) EUROCRYPT 2010. LNCS, vol. 6110, pp. 673-692. Springer, Heidelberg (2010)

26. MacKenzie, P.D., Reiter, M.K., Yang, K.: Alternatives to non-malleability: Definitions, constructions, and applications (extended abstract). In: Naor, M. (ed.) TCC 2004. LNCS, vol. 2951, pp. 171-190. Springer, Heidelberg (2004)

27. Myers, S., Shelat, A.: Bit encryption is complete. In: 50th FOCS, pp. 607-616. IEEE Computer Society Press (October 2009)

28. Pass, R., Shelat, A., Vaikuntanathan, V.: Construction of a non-malleable encryption scheme from any semantically secure one. In: Dwork, C. (ed.) CRYPTO 2006. LNCS, vol. 4117, pp. 271-289. Springer, Heidelberg (2006)

29. Peikert, C., Waters, B.: Lossy trapdoor functions and their applications. In: Ladner, R.E., Dwork, C. (eds.) 40th ACM STOC, pp. 187-196. ACM Press (May 2008)

30. Rackoff, C., Simon, D.R.: Non-interactive zero-knowledge proof of knowledge and chosen ciphertext attack. In: Feigenbaum, J. (ed.) CRYPTO 1991. LNCS, vol. 576, pp. 433-444. Springer, Heidelberg (1992)

31. Rosen, A., Segev, G.: Chosen-ciphertext security via correlated products. In: Reingold, O. (ed.) TCC 2009. LNCS, vol. 5444, pp. 419-436. Springer, Heidelberg (2009)

32. Wee, H.: Efficient chosen-ciphertext security via extractable hash proofs. In: Rabin, T. (ed.) CRYPTO 2010. LNCS, vol. 6223, pp. 314-332. Springer, Heidelberg (2010) 